\title{
SINGULAR BOUNDARY METHOD IN A FREE VIBRATION ANALYSIS OF COMPOUND LIQUID-FILLED SHELLS
}

\author{
VASYL I. GNITKO ${ }^{1}$, KYRYL G. DEGTYARIOV ${ }^{1}$, ARTEM O. KARAIEV $^{2 *} \&$ ELENA A. STRELNIKOVA $^{1,2 \dagger}$ \\ ${ }^{1}$ A.N. Podgorny Institute of Mechanical Engineering Problems of the Ukrainian Academy of Sciences, Ukraine \\ ${ }^{2}$ V.N. Karazin Kharkiv National University, Ukraine
}

\begin{abstract}
A new numerical approach is proposed to address problems of free liquid vibrations in axisymmetric compound rigid shells using a singular boundary method. The liquid is supposed to be perfect and incompressible, and its flow is irrotational so the liquid velocity can be presented as a potential gradient. The approximation of a small fluid surface elevation is used, and the free surface function is presented as a sum of the fluid-filled shell height and the small elevation function. A series expansion of the potential function about stationary states is used. To find the stationary states, an eigenvalue problem is formulated. Eigenvalues and eigenvectors are obtained using the singular boundary method with the origin intensity factor as the singular integral over the singular boundary element. The numerical results for free vibration analysis of cylindrical shells, obtained by singular boundary method and direct boundary element methods, are compared. Different compound rigid shells are considered in numerical simulations of free liquid vibrations.
\end{abstract}

Keywords: free vibrations, axisymmetric problems, potential theory, singular boundary method, compound shells, surface tension, original intensity factor.

\section{INTRODUCTION}

The boundary-type methods are widely used in different research areas, such as potential theory, elasticity and electromagnetic theories, heat transfer, fracture mechanics, fluid dynamics, biomedicine [1], [2]. Compared with domain-type techniques, such as finite elements methods, boundary element methods (BEM) have certain advantages as stability of calculation processes, high accuracy, fast convergence, and boundary only discretization, i.e., reducing the dimension of problems by one and so essentially shortening computer time and storage. However, the mathematical tools used in boundary element formulations often seem to be unfamiliar and difficult to customers, particularly at numerical simulations based on evaluating singular and hypersingular integrals. In recent years, the alternative boundary-type techniques are developed such as mesh-free and integration-free approaches [3]-[5]. Both of them use the method of fundamental solution (MFS) with prescribed source point locations as basic concept. The MFS is one of the collocation-based boundary-type, mesh-free method. In order to avoid the singularity of fundamental solutions, the source points are located on fictitious boundaries inside or outside the calculation domain relatively to internal or external boundary value problems. This method is mathematically simple, because it does not require thorough gridding and avoids calculation of complicated singular integrals. But the main problem of MFS is in proper choosing the set of source points. The new concept of origin intensity factors (OIFs) to isolate the singularity of fundamental solutions is introduced in singular boundary method (SBM) [3], [6]. In this method the source points are placed on the boundaries in coincidence with collocation points. The OIF concept is first proposed in Chen et al. [3], where an inverse interpolation technique is developed with using the set of sample points

\footnotetext{
* ORCID: https://orcid.org/0000-0003-3176-8496

† ORCID: https://orcid.org/0000-0003-0707-7214
} 
placed inside the domain. In recent research the SBM is successfully applied to potential [7], heat conduction [8], acoustic stokes flow [9], and biharmonic [10] problems.

In this paper, the problem of free liquid vibrations is formulated and solved by using the direct and indirect traditional BEM, and with singular boundary method. Here mixed boundary value problems are considered.

\section{PROBLEM STATEMENT OF LIQUID VIBRATIONS IN RIGID SHELLS OF REVOLUTION}

\subsection{Boundary value problem}

The problem of free harmonic axisymmetric liquid vibrations in rigid compound shells of revolution is considered. We designate a moistened shell surface by $S_{1}$, and a free surface by $\mathrm{S}_{0}$. Suppose the Cartesian coordinate system $0 x y z$ is connected with the shell, the liquid free surface $S_{0}$ coincides with the plane $z=H$ at the state of rest, Fig. 1.
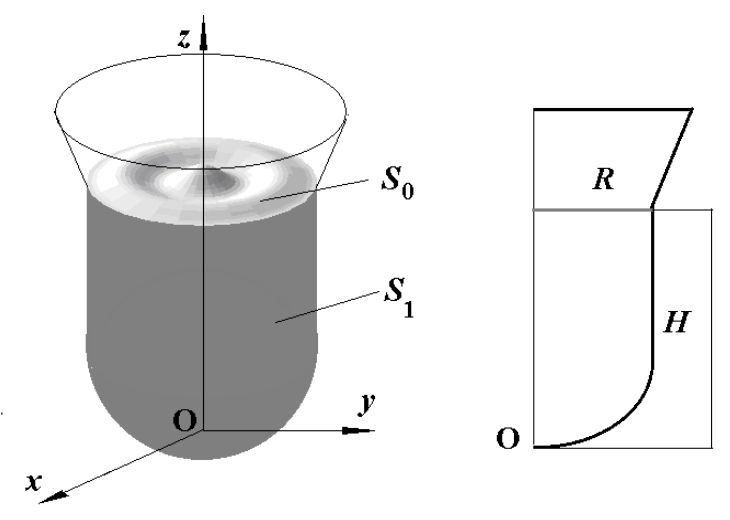

Figure 1: Liquid-filled shell of revolution and its draft.

Fluid velocity $\mathrm{v}$ inside inelastic shells of revolutions satisfies Euler's system of equations for an ideal fluid [11]:

$$
\frac{\partial \rho}{\partial t}+\operatorname{div} \rho \mathbf{v}=0, \quad \rho\left(\frac{\partial \mathbf{v}}{\partial t}+(\mathbf{v}, \nabla) \mathbf{v}\right)=-\nabla P+\mathbf{F} .
$$

Here, $\mathrm{F}$ is an external force acting on the dynamical system. Let $\mathrm{g}$ be the gravity acceleration, $\rho_{l}$ is for the liquid density, and $\mathrm{r}$ is a radius-vector of the point inside the liquid domain. Suppose that the liquid is incompressible, so $\rho_{l}=$ const, and the fluid motion is irrotational so there exist the velocity potential $\mathbf{v}=\nabla \varphi$. Assume that the fluid is located in the gravity field, and the only external force acting on the system is the gravity force $\mathbf{F}=\rho_{l} \mathbf{g}$.

With these assumptions, Euler's system (1) is transformed to [11]:

$$
\Delta \varphi=0, \quad \nabla\left(\frac{\partial \varphi}{\partial t}+\frac{1}{2}|\nabla \varphi|^{2}+\frac{1}{\rho_{l}} P-(\mathbf{g}, \mathbf{r})\right)=0 .
$$


Last equality in eqn (2) leads to the Bernoulli equation:

$$
\frac{\partial \varphi}{\partial t}+\frac{1}{2}|\nabla \varphi|^{2}+\frac{1}{\rho} P-(\mathbf{g}, \mathbf{r})=C(t)
$$

The constant $C(t)$ on the right-hand side of eqn (3) can be absorbed in the potential. We can set this constant as the derivative of another function $B(t)$ :

$$
C(t)=\frac{d B(t)}{d t}, \quad \varphi(\mathbf{r}, t) \rightarrow \varphi(\mathbf{r}, t)-B(t)
$$

The gravity acceleration is directed against $\mathrm{O} z$ axis, so the Bernoulli equation takes the following form:

$$
\frac{\partial \varphi}{\partial t}+\frac{1}{2}|\nabla \varphi|^{2}+\frac{1}{\rho_{l}} P+g z=0
$$

The Laplace equation for the potential $\varphi$ is the governing equation of the fluid motion. When solution of this equation is received, then the fluid velocity $\mathrm{v}$ can be obtained as the gradient of the velocity potential $\varphi$, and the pressure $p$ can be defined from Bernoulli's equation (5).

So it is necessary to formulate the boundary conditions for the Laplace equation. The shell walls are inelastic, so the velocity $\mathrm{v}$ has only tangential component, and [12]:

$$
\left.\frac{\partial \varphi}{\partial \mathbf{n}}\right|_{S_{1}}=0
$$

where $\mathrm{n}$ is an external unit normal to the shell wetted surface.

There are some difficulties with the free surface boundary conditions. Firstly, introduce an unknown function $\zeta$ that describes the $z$-coordinate of the free surface. Any point on the free surface is defined by its radius-vector $r$ that in the axisymmetric case has two components, $\mathbf{r}=\mathbf{r}(\rho(t), \zeta(\rho, t))$.

To define Euler's velocity in this point, we need to take the following total time derivative:

$$
\mathbf{v}(\mathbf{r}, t)=\frac{d \mathbf{r}}{d t}=\left(\frac{d \rho}{d t}, \frac{d \zeta}{d t}\right)
$$

This procedure gives two following identities for the velocity on the free surface:

$$
\left.v_{\rho}\right|_{S_{0}}=\frac{d \rho}{d t}=\left.\frac{\partial \varphi}{\partial \rho}\right|_{S_{0}},\left.v_{z}\right|_{S_{0}}=\frac{d \zeta}{d t}=\frac{\partial \zeta}{\partial t}+\frac{\partial \zeta}{\partial \rho} \frac{d \rho}{d t}=\frac{\partial \zeta}{\partial t}+v_{\rho} \frac{\partial \zeta}{\partial \rho}=\frac{\partial \zeta}{\partial t}+\left.\frac{\partial \varphi}{\partial \rho}\right|_{S_{0}} \frac{\partial \zeta}{\partial \rho}=\left.\frac{\partial \varphi}{\partial z}\right|_{S_{0}} .
$$

So, the time derivative of the free-surface function $\zeta(\rho, t)$ satisfies the next identity:

$$
\frac{\partial \zeta}{\partial t}=\left.\frac{\partial \varphi}{\partial z}\right|_{S_{0}}-\left.\frac{\partial \varphi}{\partial \rho}\right|_{S_{0}} \frac{\partial \zeta}{\partial \rho}
$$

Now we define a unit outward normal to the free surface as below: 


$$
\left.\mathbf{n}\right|_{S_{0}}=\left(-\frac{\partial \zeta}{\partial \rho}, 1\right) / \sqrt{1+\zeta_{\rho}^{\prime 2}} .
$$

From here we get relationship between Euler's and Lagrange's coordinates on the free surface:

$$
\frac{\partial \zeta}{\partial t}==\frac{1}{\sqrt{1+\zeta_{\rho}^{\prime 2}}}\left(\left.n_{\rho} \frac{\partial \varphi}{\partial \rho}\right|_{S_{0}}+\left.n_{z} \frac{\partial \varphi}{\partial z}\right|_{S_{0}}\right)=\left.\frac{1}{\sqrt{1+\zeta_{\rho}^{\prime 2}}} \frac{\partial \varphi}{\partial \mathbf{n}}\right|_{S_{0}} .
$$

Combining these conditions to get an equation with the potential function only, we need to take a time derivative from Bernoulli eqn (5) on the free surface:

$$
\left.\frac{\partial^{2} \varphi}{\partial t^{2}}\right|_{S_{0}}+\left.\frac{1}{2} \frac{\partial|\nabla \varphi|^{2}}{\partial t}\right|_{S_{0}}+\left.\frac{1}{\rho_{l}} \frac{\partial P}{\partial t}\right|_{S_{0}}=0
$$

As the pressure on the free surface is equal to atmospheric one, its time derivative can be expressed in the following manner:

$$
\left.\frac{\partial P}{\partial t}\right|_{S_{0}}+\left.\frac{\partial P}{\partial z}\right|_{S_{0}} \frac{\partial \zeta}{\partial t}=\frac{\partial P_{0}}{\partial t}=\left.0 \Rightarrow \frac{\partial P}{\partial t}\right|_{S_{0}}=-\left.\frac{\partial P}{\partial z}\right|_{S_{0}} \frac{\partial \zeta}{\partial t}=-\left.\left.\frac{\partial P}{\partial z}\right|_{S_{0}} \frac{1}{\sqrt{1+\zeta_{\rho}^{\prime 2}}} \frac{\partial \varphi}{\partial \mathbf{n}}\right|_{S_{0}} .
$$

The second component of the pressure gradient is received from second Euler's equation using eqn (5) as:

$$
-\frac{1}{\rho_{l}} \frac{\partial P}{\partial z}=\frac{\partial v_{z}}{\partial t}+\frac{1}{2}\left(\frac{\partial v_{\rho}^{2}}{\partial z}+\frac{\partial v_{z}^{2}}{\partial z}\right)+g=\frac{\partial^{2} \varphi}{\partial t \partial z}+\frac{1}{2}\left(\frac{\partial}{\partial z}\left(\frac{\partial \varphi}{\partial \rho}\right)^{2}+\frac{\partial}{\partial z}\left(\frac{\partial \varphi}{\partial z}\right)^{2}\right)+g .
$$

Thus, we receive the next relation:

$$
\left.\frac{\partial^{2} \varphi}{\partial t^{2}}\right|_{S_{0}}+\left.\frac{1}{2} \frac{\partial|\nabla \varphi|^{2}}{\partial t}\right|_{S_{0}}+\left.\left.\left(\frac{\partial^{2} \varphi}{\partial t \partial z}+\frac{1}{2}\left(\frac{\partial}{\partial z}\left(\frac{\partial \varphi}{\partial \rho}\right)^{2}+\frac{\partial}{\partial z}\left(\frac{\partial \varphi}{\partial z}\right)^{2}\right)+g\right)\right|_{S_{0}} \cdot \frac{1}{\sqrt{1+\zeta_{\rho}^{\prime 2}}} \frac{\partial \varphi}{\partial \mathbf{n}}\right|_{S_{0}}=0
$$

Considering the small vibrations one can neglect the velocity's squares. In assumption that the pressure gradient along $z$-axis depends only on the gravitational field, the velocity alterations haven't large influence on pressure. So, the linear boundary condition on the free surface takes the following form:

$$
\left.\frac{\partial^{2} \varphi}{\partial t^{2}}\right|_{S_{0}}+\left.g \frac{\partial \varphi}{\partial \mathbf{n}}\right|_{S_{0}}=0
$$

We have got the complete boundary and initial conditions for the dynamical system, and finally the boundary value problem for the Laplace equation:

$$
\Delta \varphi=0 ;\left.\quad \frac{\partial^{2} \varphi}{\partial t^{2}}\right|_{S_{0}}+\left.g \frac{\partial \varphi}{\partial \mathbf{n}}\right|_{S_{0}}=0 ;\left.\quad \frac{\partial \varphi}{\partial \mathbf{n}}\right|_{S_{1}}=0 ; \quad \frac{\partial \zeta}{\partial t}=\left.\frac{\partial \varphi}{\partial \mathbf{n}}\right|_{S_{0}} .
$$


The following solvability condition needs to be added [12]:

$$
\int_{S_{0}} \frac{\partial \varphi}{\partial \mathbf{n}} d S_{0}=0
$$

It would be noted that the formulation of the boundary value problem in eqn (17) is received as the result of linearization of free surface boundary conditions and can be considered as a first approximation of nonlinear sloshing in the shells.

\subsection{Stationary states}

To find stationary states of system (17), we separate the velocity potential into spatial and temporal functions [13]:

$$
\varphi(\mathbf{r}, t)=\psi(\mathbf{r}) e^{i \omega t}, \quad i^{2}=-1 .
$$

Using eqn (19), the following boundary value problem for the function $\psi$ is received:

$$
\Delta \psi=0,\left.\quad \frac{\partial \psi}{\partial \mathbf{n}}\right|_{S_{0}}=\left.\frac{\omega^{2}}{g} \psi\right|_{S_{0}},\left.\quad \frac{\partial \psi}{\partial \mathbf{n}}\right|_{S_{1}}=0, \iint_{S_{0}} \frac{\partial \psi}{\partial \mathbf{n}} d S_{0}=0 .
$$

These relations lead to an eigenvalue problem. Its eigenvalues $\omega_{k}$ are natural frequencies of sloshing, and its eigenvectors $\psi_{k}(\mathbf{r})$ are sloshing natural modes. Each of these eigenvectors is the solution of system (20), and every velocity potential can be expanded in terms of potentials of the stationary states as below [13]:

$$
\varphi(\mathbf{r}, t)=\sum_{k=1}^{\infty} \alpha_{k} \varphi_{k}(\mathbf{r}, t)=\sum_{k=1}^{\infty} \alpha_{k} \psi_{k}(\mathbf{r}) e^{i \omega_{k} t}
$$

Expression for the surface function $\zeta(\rho, t)$ is obtained from the Bernoulli equation on the free surface, namely we have:

$$
\zeta(\rho, t)=-\left.\frac{1}{g} \frac{\partial \varphi}{\partial t}\right|_{S_{0}}=-\left.\frac{1}{g} \frac{\partial \varphi}{\partial t}\right|_{z=\zeta(\rho, t)}=-\frac{i}{g} \sum_{k=1}^{\infty} \alpha_{k} \omega_{k} \varphi_{k}(\rho, \zeta(\rho, t), t) .
$$

To find unknown coefficients in (22) we need to know the initial shape of the surface function:

$$
\zeta\left(\rho, t_{0}\right)=\zeta_{0}(\rho)=-\frac{i}{g} \sum_{k=1}^{\infty} \alpha_{k} \omega_{k} \varphi_{k}\left(\rho, \zeta_{0}(\rho), t_{0}\right) .
$$

Using the approximation of small fluid surface elevation, one can present the free surface function as the sum of the fluid-filled shell height and a small elevation function $\varepsilon(\rho, t)$ :

$$
\zeta(\rho, t)=H+\varepsilon(\rho, t)
$$


We can expand $\varphi_{k}(\rho, z, t)$ into Taylor series near the unperturbed free surface state considering constant values of $\rho$ and $t$ :

$$
\varphi_{k}(\rho, z, t)=\varphi_{k}(\rho, H, t)+\left.\frac{\partial \varphi_{k}}{\partial z}\right|_{z=H}(z-H)+\left.\frac{1}{2} \frac{\partial^{2} \varphi_{k}}{\partial z^{2}}\right|_{z=H}(z-H)^{2}+\cdots
$$

It is possible to retain the only linear members in expansion (25) in the point $z=\zeta(\rho, t)$ due to the small values of $\varepsilon^{2}(\rho, t)$ and higher terms:

$$
\varphi_{k}(\rho, \zeta(\rho, t), t)=\varphi_{k}(\rho, H, t)+\left.\frac{\partial \varphi_{k}}{\partial z}\right|_{z=H} \varepsilon(\rho, t) .
$$

Using eqn (26), we received the elevation function $\varepsilon(\rho, t)$ from eqn (21) as follows:

$$
\zeta(\rho, t)=H-\frac{g H+i \sum_{k=1}^{\infty} \alpha_{k} \omega_{k} \varphi_{k}(\rho, H, t)}{g+\left.i \sum_{k=1}^{\infty} \alpha_{k} \omega_{k} \frac{\partial \varphi_{k}}{\partial z}\right|_{z=H}} .
$$

So, we come to the spectral boundary problem on the natural sloshing modes and frequencies. Thus, the problem of liquid vibrations in gravitational field is reduced to finding the natural frequencies and basis eigenvectors.

\subsection{Eigen frequencies and eigenvectors problem}

To find our own frequencies and eigenvectors, we use a singular boundary method, but not in its classical formulation. Naturally, singular boundary methods are developed from the source density formulation. In this formulation, the unknown function and its normal derivative are presented in the next form [1]:

$$
\begin{gathered}
\psi(\xi)=\oint_{S} \sigma(\mathbf{r}) u^{*}(\xi, \mathbf{r}) d S(\mathbf{r}), \\
\frac{\partial \psi(\xi)}{\partial \mathbf{n}(\xi)}=2 \pi \sigma(\xi)+\oint_{S} \sigma(\mathbf{r}) q^{*}(\xi, \mathbf{r}) d S(\mathbf{r}) .
\end{gathered}
$$

Here, $\sigma(\mathbf{r})$ is a surface density of the unknown function $\psi(\xi)$ :

$$
u^{*}(\xi, \mathbf{r})=\frac{1}{|\xi-\mathbf{r}|}, \quad q^{*}(\xi, \mathbf{r})=\frac{\partial}{\partial \mathbf{n}(\xi)}\left[\frac{1}{|\xi-\mathbf{r}|}\right] .
$$

However, using representation (28) creates difficulties in solving the problem under consideration in corner points, because it is impossible to define correctly the normal vector in corner points in classical sense, and in vicinities of these points the potential normal derivative tends to satisfy both conditions (6) and (16). This means that the normal derivative in corner points cannot satisfy Lipschitz continuity [14] so the surface density is not continuous in these points. If we use above formulation, we need to present 
integrals in eqn (28) as partial sums, but in the vicinities of the corner points the partial sums tend to disconverge.

In the current research, authors use third Green's identity as governing equation [1]:

$$
2 \pi \psi(\xi)+\oint_{S} \psi(\mathbf{r}) q^{*}(\xi, \mathbf{r}) d S(\mathbf{r})=\oint_{S} \frac{\partial \psi(\mathbf{r})}{\partial \mathbf{n}(\mathbf{r})} u^{*}(\xi, \mathbf{r}) d S(\mathbf{r}) .
$$

We divide boundary into $N$ boundary elements; at that $m$ elements belong to the free surface and others $N-m$ belong to the shell walls. At each boundary element we select the collocation point. In doing so, we represent the surface integrals in eqn (30) as sums of integrals over surfaces $S_{i}, i=\overline{1, N}$ of each boundary element. For each collocation point the following equation is valid:

$$
2 \pi \psi\left(\mathbf{r}_{k}\right)+\sum_{i=1}^{N} \int_{S_{i}} \psi(\mathbf{r}) q^{*}\left(\mathbf{r}_{k}, \mathbf{r}\right) d S_{i}=\sum_{i=1}^{N} \int_{S_{i}} \frac{\partial \psi(\mathbf{r})}{\partial \mathbf{n}(\mathbf{r})} u^{*}\left(\mathbf{r}_{k}, \mathbf{r}\right) d S_{i} .
$$

We apply Cauchy's average theorem to eqn (31) and receive:

$$
2 \pi \psi\left(\mathbf{r}_{k}\right)+\sum_{i=1}^{N} q^{*}\left(\mathbf{r}_{k}, \mathbf{r}_{i}\right) \int_{S_{i}} \psi(\mathbf{r}) d S_{i}=\sum_{i=1}^{N} u^{*}\left(\mathbf{r}_{k}, \mathbf{r}_{i}\right) \int_{S_{i}} \frac{\partial \psi(\mathbf{r})}{\partial \mathbf{n}(\mathbf{r})} d S_{i} .
$$

Using boundary condition (16) one can obtain:

$$
\begin{aligned}
& 2 \pi \psi\left(\mathbf{r}_{k}\right)+\sum_{i=1}^{m} q^{*}\left(\mathbf{r}_{k}, \mathbf{r}_{i}\right) \int_{S_{i}} \psi(\mathbf{r}) d S_{i}+\sum_{i=m+1}^{N} q^{*}\left(\mathbf{r}_{k}, \mathbf{r}_{i}\right) \int_{S_{i}} \psi(\mathbf{r}) d S_{i} \\
& =\frac{\omega^{2}}{g} \sum_{i=1}^{m} u^{*}\left(\mathbf{r}_{k}, \mathbf{r}_{i}\right) \int_{S_{i}} \psi(\mathbf{r}) d S_{i} .
\end{aligned}
$$

Let us introduce the following denominations:

$$
\beta_{0 i}=\int_{S_{i}} \psi(\mathbf{r}) d S_{i} \text { if } S_{i} \in S_{0}, \quad \beta_{1 i}=\int_{S_{i}} \psi(\mathbf{r}) d S_{i} \text { if } S_{i} \in S_{1} .
$$

Then we can express $\beta_{1 i}$ through $\beta_{0 i}$ as:

$$
\left(\frac{2 \pi \delta_{i k}}{s_{k}}+Q_{i k}^{* 11}\right) \beta_{1 k}=\left(\frac{\omega^{2}}{g} U_{i k}^{* 10}-Q_{i k}^{* 10}\right) \beta_{0 k},
$$

where $s_{k}=\int_{S_{k}} d S_{k}, Q_{i k}^{* \alpha \beta}=q^{*}\left(\mathbf{r}_{i}, \mathbf{r}_{k}\right)$ and $U_{i k}^{* \alpha \beta}=u^{*}\left(\mathbf{r}_{i}, \mathbf{r}_{k}\right), \alpha, \beta=0$ for points on the free surface, and $\alpha, \beta=1$ for side wall points, namely:

$$
\begin{aligned}
& \alpha=0, \beta=0 \Rightarrow \mathbf{r}_{i} \in S_{0}, \mathbf{r}_{k} \in S_{0}, \alpha=0, \beta=1 \Rightarrow \mathbf{r}_{i} \in S_{0}, \mathbf{r}_{k} \in S_{1}, \\
& \alpha=1, \beta=0 \Rightarrow \mathbf{r}_{i} \in S_{1}, \mathbf{r}_{k} \in S_{0}, \alpha=1, \beta=1 \Rightarrow \mathbf{r}_{i} \in S_{1}, \mathbf{r}_{k} \in S_{1} .
\end{aligned}
$$

Thus, the system to determine the eigenvalues and eigenvectors using singular boundary method is received as following: 


$$
\begin{aligned}
& \left(\frac{2 \pi \delta_{i k}}{s_{k}}+Q_{i k}^{* 00}-Q_{i l}^{* 01}\left(\frac{2 \pi \delta_{l p}}{s_{p}}+Q_{l p}^{* 11}\right)^{-1} Q_{p k}^{* 10}\right) \beta_{0 k} \\
& =\frac{\omega^{2}}{g}\left(U_{i k}^{* 00}-Q_{i l}^{* 01}\left(\frac{2 \pi \delta_{l p}}{s_{p}}+Q_{l p}^{* 11}\right)^{-1} U_{p k}^{* 10}\right) \beta_{0 k}
\end{aligned}
$$

In equations beforehand, Einstein's summation convention is applied.

\subsection{Origin intensity factors}

It is ascertained that the diagonal elements of matrixes $Q_{i k}^{* \alpha \beta}$ and $U_{i k}^{* \alpha \beta}$ cannot be defined in the singular points as Green's function and its normal derivative. To overcome this difficulty, we use the following presentation of diagonal elements as average values of Green's function and its normal derivative:

$$
U_{k k}^{* \alpha \beta}=\frac{\int_{S_{k}} u^{*}\left(\mathbf{r}_{k}, \mathbf{r}\right) d S_{k}}{s_{k}}, Q_{k k}^{* \alpha \beta}=\frac{\int_{S_{k}} q^{*}\left(\mathbf{r}_{k}, \mathbf{r}\right) d S_{k}}{s_{k}} .
$$

These expressions are called origin intensity factors in singular boundary methods.

In the axisymmetric case, we can calculate surface integrals as curve integrals of Green's function integrated by the angle variable [15]. Green's function and its normal derivative, integrated by the angle variable, are expressed in terms of complete elliptic integrals of the first and second kind [15], [16] using the following formulae [17]

$$
\begin{gathered}
\iint_{S_{k}} \frac{\partial}{\partial \mathbf{n}}\left(\frac{1}{\left|\mathbf{r}_{k}-\mathbf{r}\right|}\right) d S_{k}=\int_{\Gamma_{k}} \Theta\left(\mathbf{r}_{k}, \mathbf{r}\right) \rho(z) d \Gamma_{k}, \quad \iint_{S_{k}} \frac{1}{\left|\mathbf{r}_{k}-\mathbf{r}\right|} d S_{k}=\int_{\Gamma_{k}} \Phi\left(\mathbf{r}_{k}, \mathbf{r}\right) \rho(z) d \Gamma_{k}, \\
\Theta\left(\mathbf{r}_{k}, \mathbf{r}\right)=\frac{4}{\sqrt{a+b}}\left\{\frac{1}{2 r}\left[\frac{\rho^{2}-\rho_{k}^{2}+\left(z_{k}-z\right)^{2}}{a-b} E(m)-K(m)\right] n_{r}+\frac{z_{k}-z}{a-b} E(m) n_{z}\right\}, \\
\Phi\left(\mathbf{r}_{k}, \mathbf{r}\right)=\frac{4}{\sqrt{a+b}} K(m), \quad \mathrm{E}(k)=\int_{0}^{\pi / 2} \sqrt{1-k^{2} \sin ^{2} \theta} d \theta, K(m)=\int_{0}^{\pi / 2} \frac{d \theta}{\sqrt{1-m^{2} \sin ^{2} \theta}}, \\
a=\rho^{2}+\rho_{k}^{2}+\left(z-z_{k}\right)^{2}, \quad b=2 \rho \rho_{k}, \quad m^{2}=\frac{2 b}{a+b} .
\end{gathered}
$$

The complete elliptic integral of the second kind $K(m)$ can be presented in the next form near singular points:

$$
K(m)=\frac{2}{\pi} \ln \frac{1}{m^{\prime}}+\frac{4}{\pi} \int_{0}^{1} \frac{1}{\sqrt{\left(1-x^{2}\right)\left(1-m^{\prime 2} x^{2}\right)}} \ln \frac{1}{x} d x, m^{\prime 2}=1-m^{2} .
$$

So, evaluating the original intensity factors is reduced to calculation of integrals with logarithmic singularity. To treat with this singularity, we use quadrature formulae with quadrature nodes in the roots of orthogonal polynomials with the logarithmic weight [1] and apply the technique described in Gnitko et al. [17]. 


\section{NUMERICAL RESULTS}

The problems of sloshing in rigid shells of revolution are solved using boundary element method and singular boundary method for comparison. Cylindrical, cylindrical-spherical, and cylindrical-conic shells are under consideration, Fig. 2.
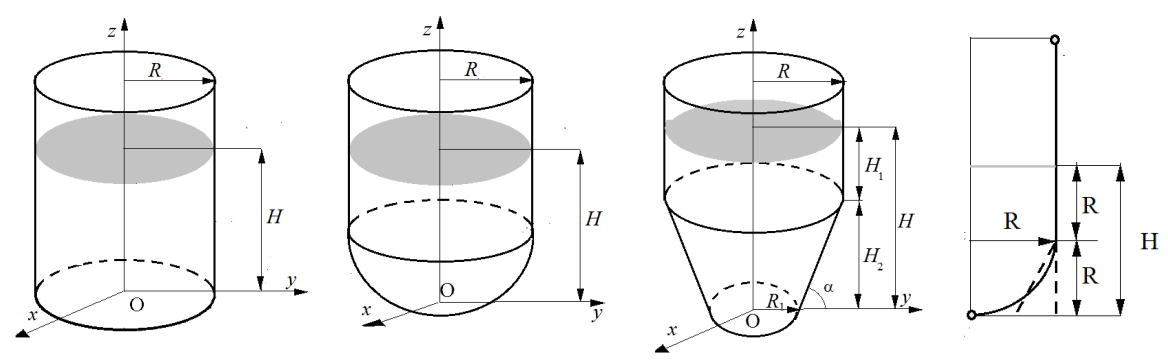

Figure 2: Liquid-filled shells of revolution.

For all shells we suppose that $R=1$ and $H=2$. The height of cylindrical parts for cylindrical-conic and cylindrical-spherical shells is equal to $R$. For the cylindrical-conic shell, we consider $R_{1}=0.5 R$.

Axisymmetric vibrations of liquid are studied. For cylindrical shell, comparison is done between numerical BEM and SBM results, and the following analytical solution [18]:

$$
\frac{\omega_{k}^{2}}{g}=\frac{\mu_{k}}{R} \tanh \left(\frac{\mu_{k} H}{R}\right), \quad \psi_{k}=J_{0}\left(\mu_{k} \frac{\rho}{R}\right) \cosh \left(\frac{\mu_{k} z}{R}\right) .
$$

Here values $\mu_{k}$ are the roots of the equations

$$
\frac{d J_{0}(x)}{d x}=0
$$

where $J_{0}(x)$ is the Bessel function of the first kind.

Results of comparison with analytical solution (41) for two methods are shown in Table 1 for different numbers $m$ of boundary elements on the free surface. Here, the errors (maximal difference between analytical and numerical solutions) for modes $k=\overline{0,4}$ are presented.

In the BEM simulation, the one-dimensional axisymmetrical formulation is in use [15], [16], and the SBM is described beforehand. The equal numbers of elements on the free surface $(\mathrm{m})$ and on wetted parts $(3 \mathrm{~m})$ of the shell are applied both in the BEM and SBM.

Analyzing results of Table 1, one can conclude that both methods give errors less than $1 \%$. The results obtained by the boundary element method are more accurate, but the singular boundary method has essential advantages in CPU time calculations.

The modes of vibrations for cylindrical, cylindrical-conic, cylindrical-spherical shells are shown in Figs 3, 4, and 5, respectively. Solid lines present modes of vibrations obtained using the boundary element method, and dashed lines present the singular boundary method's solutions.

It should be noted that all vibration modes demonstrate the Bessel-like behaviour. For all shells of revolution considered in this study, the liquid frequencies of axisymmetric vibrations are very close for each mode. 
Table 1: Comparison between analytical and numerical solutions of BEM and SBM.

\begin{tabular}{|c|c|c|c|c|c|c|c|}
\hline \multirow{2}{*}{$\mathrm{m}$} & & $\begin{array}{c}\text { 0th mode } \\
\text { error }\end{array}$ & $\begin{array}{c}\text { 1st mode } \\
\text { error }\end{array}$ & $\begin{array}{c}\text { 2nd mode } \\
\text { error }\end{array}$ & $\begin{array}{c}\text { 3rd mode } \\
\text { error }\end{array}$ & $\begin{array}{c}\text { 4th mode } \\
\text { error }\end{array}$ & $\begin{array}{c}\text { CPU } \\
\text { time }\end{array}$ \\
\hline \multirow{2}{*}{20} & BEM & $4.2054 \mathrm{e}-07$ & 0.0191 & 0.0094 & 0.0115 & 0.0228 & 0.14 \\
\cline { 2 - 8 } & SBM & 0.0033 & 0.0907 & 0.0277 & 0.0250 & 0.0417 & 0.043 \\
\hline \multirow{2}{*}{75} & BEM & $2.2917 \mathrm{e}-07$ & $3.9247 \mathrm{e}-04$ & 0.0010 & 0.0020 & 0.0054 & 1.952 \\
\cline { 2 - 9 } & SBM & $9.1500 \mathrm{e}-04$ & 0.0058 & 0.0089 & 0.0122 & 0.0247 & 0.474 \\
\hline \multirow{2}{*}{150} & BEM & $1.7993 \mathrm{e}-07$ & $1.3658 \mathrm{e}-04$ & $6.45 \mathrm{e}-04$ & 0.0017 & 0.0018 & 8 \\
\cline { 2 - 9 } & SBM & $4.5902 \mathrm{e}-04$ & 0.0038 & 0.0097 & 0.0186 & 0.0151 & 2 \\
\hline \multirow{2}{*}{400} & BEM & $1.4327 \mathrm{e}-07$ & $2.4538 \mathrm{e}-05$ & $7.98 \mathrm{e}-05$ & $1.80 \mathrm{e}-04$ & $1.50 \mathrm{e}-04$ & 82 \\
\cline { 2 - 8 } & SBM & $1.7552 \mathrm{e}-04$ & 0.0018 & 0.0032 & 0.0049 & 0.0032 & 29 \\
\hline
\end{tabular}

\section{CONCLUSION}

The variant of singular boundary method is proposed with a new calculation scheme for the origin intensity factors. The presentation of diagonal elements as average values of Green's function and its normal derivative is used, and the technique is elaborated for numerical estimation of the diagonal elements based on quadrature formulae with nodes in the roots of orthogonal polynomials with the logarithmic weight. This study demonstrates the applicability of the SBM to problems of liquid sloshing in rigid shells of revolution with arbitrary meridians. The emphasis is done to possibility of using SBM for fluid domains with corner points. Numerical experiments proved that the results obtained with proposed SBM are in good agreement with BEM for axisymmetric problems of liquid vibrations. The singular boundary method has the essential advantage in CPU time calculations, does not require thorough gridding and avoids calculation of complicated singular integrals. The proposed scheme of SBM reveals new possibilities for the analysis and modeling the linear and nonlinear process of the liquid movement in different reservoirs.

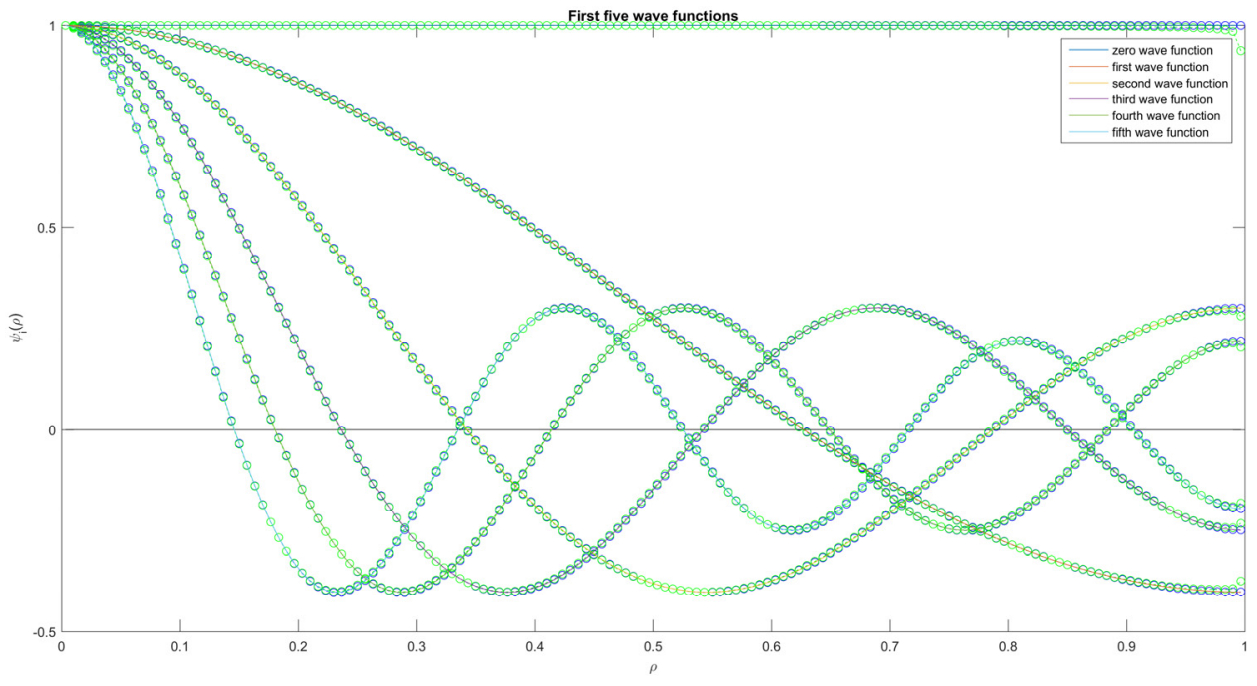

Figure 3: First five eigenmodes for cylindrical shell using BEM and SBM. 


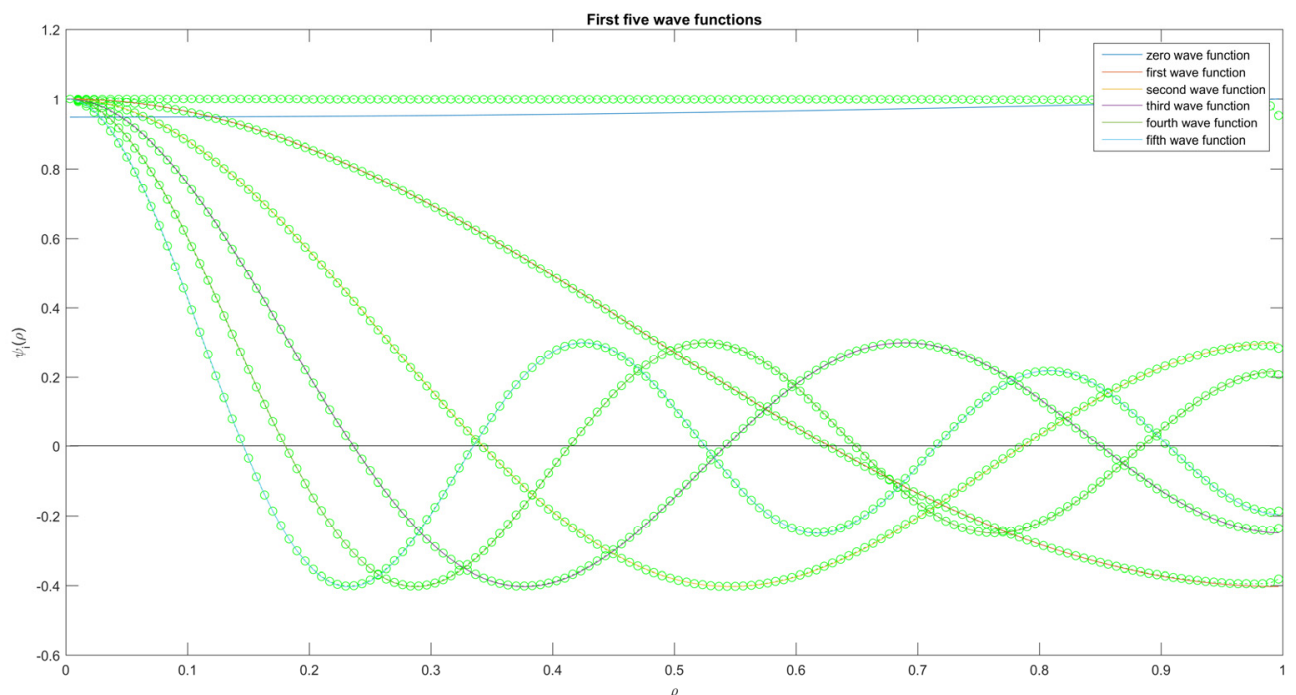

Figure 4: First five eigenmodes for cylindrical-spherical shell using BEM and SBM.

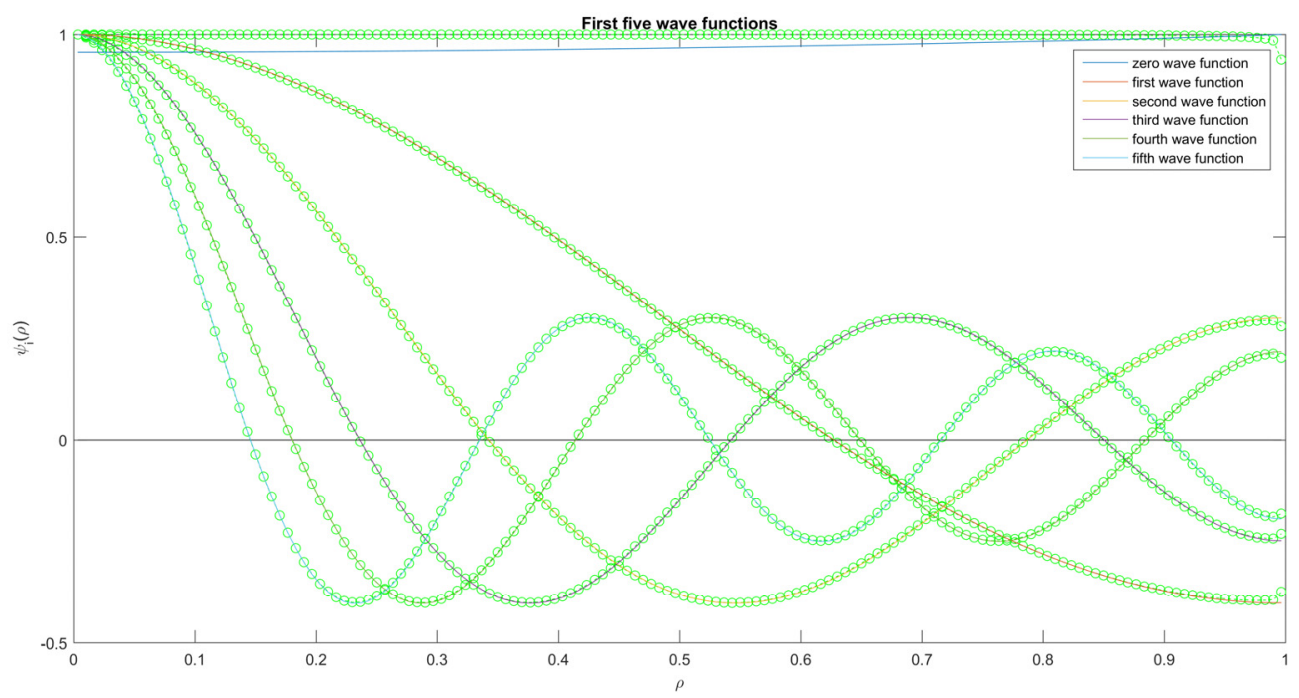

Figure 5: First five eigenmodes for cylindrical-conic shell using BEM and SBM.

\section{ACKNOWLEDGEMENTS}

Financial support for the project from STCU under grant No. 6247 is gratefully acknowledged. The authors would also like to thank our foreign collaborator, Professor Alexander Cheng, University of Mississippi, USA, for his constant support and interest to our research. 


\section{REFERENCES}

[1] Brebbia, C.A., Telles, J.C.F. \& Wrobel, L.C., Boundary Element Techniques: Theory and Applications in Engineering. Springer-Verlag, 1984.

[2] Katsikadelis, J.T., The Boundary Element Method for Engineers and Scientists, 2nd ed., Academic Press, Elsevier: Oxford, 2016.

[3] Chen, W., Zhang, J.Y. \& Fu, Z.J., Singular boundary method for modified Helmholtz equations. Eng. Anal. Bound. Elem., 44, pp. 112-119, 2014.

[4] Chen, W. \& Wang, F.Z., A method of fundamental solutions without fictitious boundary. Engineering Analysis with Boundary Elements, 34, pp. 530-532, 2010.

[5] Kleefeld, A. \& Pieronekz, L., The method of fundamental solutions for computing acoustic interior transmission eigenvalues. Inverse Problems, 34(3), 2018.

[6] Sun, L., Chen, W. \& Cheng, A.H.-D., Evaluating the origin intensity factor in the singular boundary method for three-dimensional dirichlet problems. Advances in Applied Mathematics and Mechanics, 9(6), pp. 1289-1311, 2017.

[7] Gu, Y. \& Chen, W., Infinite domain potential problems by a new formulation of singular boundary method. Applied Mathematical Modelling, 37(4), pp. 1638-1651, 2013.

[8] Gu, Y., Chen, W., Zhang, C. \& He, X., A meshless singular boundary method for three-dimensional inverse heat conduction problems in general anisotropic media. International Journal of Heat and Mass Transfer, 84, pp. 91-102, 2015.

[9] Qu, W., Chen, W., Fu, Z.-J. \& Gu, Y., Fast multipole singular boundary method for Stokes flow problems. Mathematics and Computers in Simulation, 146, pp. 57-69, 2017.

[10] Yang, C. \& Li, X., Meshless singular boundary methods for biharmonic problems. Engineering Analysis with Boundary Elements, 56, pp. 39-48, 2015.

[11] Landau, L.D. \& Lifshitz, E.M., Fluid Dynamics, Pergamon Press, 1987.

[12] Gnitko, V., Degtyariov, K., Naumenko, V. \& Strelnikova, E., BEM and FEM analysis of the fluid-structure Interaction in tanks with baffles. Int. Journal of Computational Methods and Experimental Measurements, 5(3), pp. 317-328, 2017.

[13] Landau, L.D. \& Lifshitz, E.M., Quantum Mechanics, Pergamon Press, 1965.

[14] O'Searcoid, M., Metric Spaces, Springer, 2006.

[15] Gnitko, V., Degtyariov, K., Karaiev, A. \& Strelnikova, E., Multi-domain boundary element method for axisymmetric problems in potential theory and linear isotropic elasticity. WIT Transactions on Engineering Sciences, vol. 122, WIT Press: Southampton and Boston, pp. 13-25, 2019.

[16] Ravnik, J., Strelnikova, E., Gnitko, V., Degtyarev, K. \& Ogorodnyk, U., BEM and FEM analysis of fluid-structure interaction in a double tank. Engineering Analysis with Boundary Elements, 67, pp. 13-25, 2016.

[17] Gnitko, V., Degtyarev, K., Naumenko, V. \& Strelnikova, E., Coupled BEM and FEM Analysis of fluid-structure interaction in dual compartment tanks. International Journal of Computational Methods and Experimental Measurements, 6(6), pp. 976988, 2018.

[18] Ibrahim, R.A., Liquid Sloshing Dynamics, Cambridge University Press: Cambridge, 2005. 